\title{
Fluvastatin protects myocardial cells in mice with acute myocardial infarction through inhibiting RhoA/ROCK pathway
}

\author{
ZHENCI YI $^{1 *}$, JIAYING KE $^{2 *}$, YAOGUO WANG ${ }^{3}$ and KAIJIN CAI ${ }^{3}$ \\ ${ }^{1}$ Department of Pharmacy, The Second Affiliated Hospital of Fujian Medical University; \\ ${ }^{2}$ Department of Marine Biology, Quanzhou Normal University; ${ }^{3}$ Department of Emergency, \\ The Second Affiliated Hospital of Fujian Medical University, Quanzhou, Fujian 362000, P.R. China
}

Received September 27, 2019; Accepted November 25, 2019

DOI: $10.3892 /$ etm.2020.8413

\begin{abstract}
Protective effect of fluvastatin (Flu) on myocardial cells in mice with acute myocardial infarction (AMI) and the mechanism were explored. Forty C57B/L6 mice in similar physiological status were selected and randomly divided into sham operation (Sham) group $(n=10)$, AMI group $(n=10)$, Flu group $(\mathrm{n}=10)$ and Flu + Angiotensin II (Ang II) (Ang II) group $(n=10)$. The pathological changes in heart tissues were detected via hematoxylin and eosin (H\&E) staining, and apoptosis of myocardial cells was detected via terminal deoxynucleotidyl transferase-mediated dUTP nick end labeling (TUNEL) assay. Moreover, the expression levels of malondialdehyde (MDA) and superoxide dismutase (SOD) were determined using relevant kits, and the expression levels of Ras homolog gene family (Rho)-associated coiled-coil protein kinase 1 (ROCK1), ROCK2, B-cell lymphoma-2 (Bcl-2), Bcl-2 associated X protein (Bax) and nuclear factor- $\kappa \mathrm{B}(\mathrm{NF}-\kappa \mathrm{B})$ in the infarction region were determined using Western blotting. The infarction area in mice in Flu group was significantly smaller than that in AMI group. In AMI group, the level of MDA in the serum and infarction tissues was remarkably higher than that in Sham group $(\mathrm{P}<0.05)$, while that of SOD significantly declined $(\mathrm{P}<0.05)$. The level of MDA in Flu group was obviously lower than that in AMI group $(\mathrm{P}<0.05)$. The expression levels of Bax, $\mathrm{NF}-\kappa \mathrm{B}, \mathrm{ROCK} 1$ and ROCK2 were obviously higher in AMI group than those in Sham group, while they were obviously lower in Flu group than those in AMI group $(\mathrm{P}<0.05)$. After the Rho member A (RhoA)/ROCK pathway agonist Ang II was added, the mitigation effect of Flu on myocardial apoptosis in the infarction region in AMI mice was evidently weakened.
\end{abstract}

Correspondence to: Dr Kaijin Cai, Department of Emergency, The Second Affiliated Hospital of Fujian Medical University, 34 North Zhongshan Road, Quanzhou, Fujian 362000, P.R. China

E-mail: sdyno985@163.com

*Contributed equally

Key words: fluvastatin, myocardial cell, mouse, aute myocardial infarction, RhoA/ROCK
Flu mitigates AMI-induced myocardial apoptosis in mice, and the possible mechanism is that the inflammatory and oxidative stress responses activated and mediated by RhoA/ROCK are effectively inhibited.

\section{Introduction}

In pathogenesis of myocardial ischemia the coronary blood flow perfusion declines due to the changes in coronary circulation, thus reducing the oxygen supply to the heart and leading to abnormal myocardial energy metabolism, which often causes myocardial damage. Ischemic heart disease is one of the major diseases seriously threatening human health in today's society. Various types of angina and acute myocardial infarction (AMI) often lead to sudden death of patients, and their morbidity and mortality rates are high world-wide (1).

Apoptosis is a kind of normal physiological mechanism of the body, as well as another form of death different from necrosis. Under physiological conditions, apoptosis is involved in regulating the renewal of normal cells and clearance of abnormal cells from the body, and maintaining homeostasis. Under pathological conditions, apoptosis is related to ischemia, reperfusion injury, abnormal virus infection, transplant rejection and tumors (2-4).

Myocardial ischemia is one of the strong inducers of myocardial apoptosis (5). After MI, the central areas of infarction mainly present necrosis of myocardial cells, due to severe ischemia and hypoxia, while in the infarction border region the myocardial cells mainly present apoptosis that is reversible due to milder ischemia. Therefore, reducing myocardial apoptosis in the infarction border region and non-infarction region is of importance for reducing the damage after MI. During apoptosis, downstream effector cysteine aspartic acidspecific protease-3 (Caspase-3) will be eventually activated by endogenous and exogenous pathways (6), and Caspase-3 plays an important role in apoptosis after MI $(7,8)$. Moreover, the balance between the pro-apoptotic proteins [Bid, B-cell lymphoma-2 (Bcl-2) associated $\mathrm{X}$ protein (Bax) and Bak] and anti-apoptotic proteins (Bcl-2 and Bcl-xL) in the Bcl-2 family plays an important role in initiating the endogenous apoptotic pathway (9). Bax, induced by myocardial ischemia, can be transported to the mitochondrial outer membrane to initiate the endogenous mitochondrial apoptotic pathway. Due 
to the activated caspase cascade, the caspase- 3 precursors in the cytoplasm are activated and lysed into activated caspase-3, ultimately leading to apoptosis. However, the expression of Bcl-2 can stabilize the mitochondrial membrane and inhibit the occurrence and development of apoptosis.

The Ras homolog gene family (Rho)/Rho kinase signaling pathway is composed of Rho protein, Rho kinase, myosin phosphatase and other signaling molecules. Rho protein is a kind of small-molecule guanine nucleotidebinding protein, also known as small $\mathrm{G}$ protein, as well as a member of the Ras protein superfamily, which serves as a signal converter or molecular switch in the cellular signal transduction pathway, and acts on the cytoskeleton and its target proteins, thus exerting various biological effects. Among the Rho family members, Rho member A (RhoA) plays not only an important role in the regulation of cytoskeleton, maintenance of cellular morphology, cell migration, smooth muscle cell contraction and other cell activities, but also has a key role in various biological activities, such as smooth muscle cell proliferation, cell adhesion, platelet aggregation, contact inhibition, growth and apoptosis (10-12). During cellular metabolism, Rho protein exists in the guanine trinucleotide phosphate (GTP)- and guanine dinucleotide phosphate (GDP)-binding forms, and it induces or terminates the cellular cascade activation through mutual conversion between GDP phosphorylation and GTP dephosphorylation.

The statins, namely 3-hydroxy-3-methylglutaryl coenzyme A (HMG-CoA) reductase inhibitors, are a new type of drugs widely used to lower the cholesterol level. It has been confirmed in evidence-based medicine that in addition to the lipidlowering effect, statins also possess a series of cardiovascular protective effects, such as anti-inflammation, anti-oxidation, improvement of endothelial function, inhibition on smooth muscle proliferation, and inhibition on platelet aggregation and thrombosis $(13,14)$. Moreover, the non-lipid-lowering effects (such as anti-inflammatory, anti-oxidation and immunoregulatory effects) of fluvastatin (Flu), as a kind of classical statin, have attracted increasing attention. Existing studies have proved that Flu can improve myocardial apoptosis, ventricular remodeling and cardiac dysfunction after MI $(15,16)$, but its mechanism of action has not been clarified yet. In the present study, therefore, the mouse model of AMI was established to explore the antagonistic effect of Flu on AMI and myocardial apoptosis, and elucidate its possible mechanism through the RhoA/Rho-associated coiled-coil protein kinase 1 (ROCK1) pathway, to lay a theoretical foundation for the application of Flu in the prevention and treatment of AMI.

\section{Materials and methods}

Animals and reagents. This study was approved by the Animal Ethics Committee of Fujian Medical University Animal Center (Quanzhou, China). A total of $40 \mathrm{C} 57 \mathrm{BL} / 6 \mathrm{~J}$ mice aged 8 weeks and weighing $17.9 \pm 0.6 \mathrm{~g}$ with normal nutritional status and mental status, were provided by Laboratory Animal Center of Fujian Medical University. Bcl-2-associated X protein (Bax), Bcl-2, NF-кB, ROCK1, ROCK2 antibodies were from SigmaAldrich; Merck KGaA. TUNEL staining kit QIA33 was from Shanghai Shiyi Biotechnology Co., Ltd., and the superoxide dismutase (SOD) and malondialdehyde (MDA) test kits were from Nanjing Jiancheng Bioengineering Research Institute.

Model establishment and grouping. Before the operation, mice were weighed, intraperitoneally injected with avertin solution at a dose of $0.40-0.75 \mathrm{mg} / \mathrm{g}$ for anesthesia, and fixed on a rat plate in supine position. Then, the skin of the neck and precordial region was disinfected with $70 \%$ alcohol, and the hair was shaved. After that, the skin of the neck was scissored, and the muscle was bluntly separated, followed by tracheal intubation and connection of a small animal ventilator (positive pressure ventilation 2-3 ml/cycle, frequency 20 cycles $/ \mathrm{min}$ ). Thereafter, the skin of precordial region was scissored, the muscle was bluntly separated, and the chest wall was cut using a pair of scissors at the space between the third and fourth ribs. For 30 mice in AMI group, Flu group, Ang II group (group A), a 7-0 suture needle with suture was used to ligate the left anterior descending coronary artery (coronary artery) at 3-4 mm below the left auricle. For another 10 mice serving as Sham group (group B), the needle was passed through the left anterior descending coronary artery, but the artery was not ligated. Then, the thoracic cavity was closed along the third and fourth ribs, the chest muscles were sutured layer by layer, and finally the chest skin was continuously sewn up. After that, the chest was squeezed to remove thoracic gas to recruit the lungs, the ventilator was removed, and the neck skin was sewn up. Lastly, mice were put on a $40^{\circ} \mathrm{C}$ insulation platform for regaining consciousness.

Immunohistochemistry. Paraffin-embedded heart tissue sections were deparaffinized with xylene, dehydrated with graded alcohol, and incubated with warm deionized water containing $0.3 \% \mathrm{H}_{2} \mathrm{O}_{2}$ for $30 \mathrm{~min}$. After endogenous peroxide was eliminated, sections were blocked with serum and addeded with primary antibodies for incubation at $4^{\circ} \mathrm{C}$ overnight. The next day, sections were incubated with $\operatorname{IgG}$ antibody-HRP, and dropwise added with the mixture prepared in the biotin and $\mathrm{ABC}$ kit for culture, followed by color development with DAB for $10 \mathrm{~min}$. Thereafter, sections were counterstained with hematoxylin, washed, dehydrated and permeabilized. Lastly, sections were observed using an optical microscope.

Terminal deoxynucleotidyl transferase-mediated deoxyuridine triphosphate (dUTP)-biotin nick end labeling (TUNEL). Paraffin-embedded tissue sections were deparaffinized with xylene, dehydrated with graded alcohol, and treated with Proteinase $\mathrm{K}$ working solution for $15-30 \mathrm{~min}$ at $21-37^{\circ} \mathrm{C}$ or added with cell-permeable solution for $8 \mathrm{~min}$ of reaction. TUNEL reaction mixture was prepared; $50 \mu \mathrm{l} \mathrm{TdT}+450 \mu 1$ fluorescein-labeled dUTP solution was added and mixed in treatment group, and only $50 \mu 1$ fluorescein-labeled dUTP solution was added in negative control group. After slides were dried, $50 \mu 1$ TUNEL reaction mixture (only $50 \mu 1$ fluoresceinlabeled dUTP solution was added in negative control group) was added onto specimens, and slides were then covered with cover slips or sealing films, followed by reaction in the dark and humid chamber at $37^{\circ} \mathrm{C}$ for $60 \mathrm{~min}$. After the slides were dried, $50 \mu \mathrm{l}$ converter-POD was added onto specimens, slides were then covered with cover slips or sealing films, followed by reaction in the dark and humid chamber at $37^{\circ} \mathrm{C}$ for $30 \mathrm{~min}$. 
A

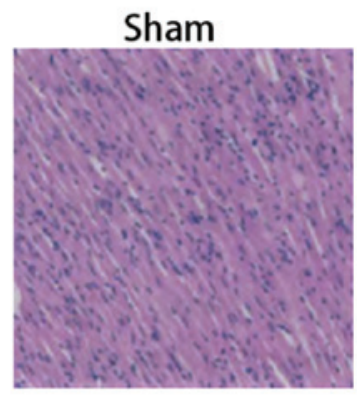

B

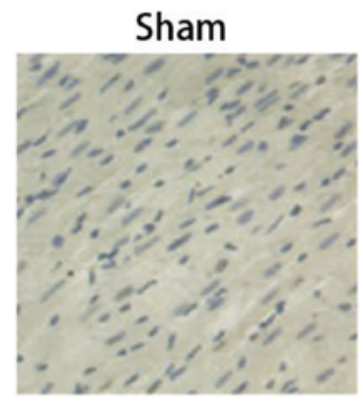

AMI

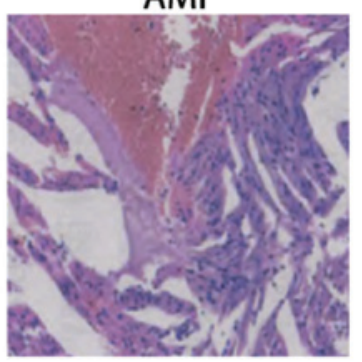

AMI

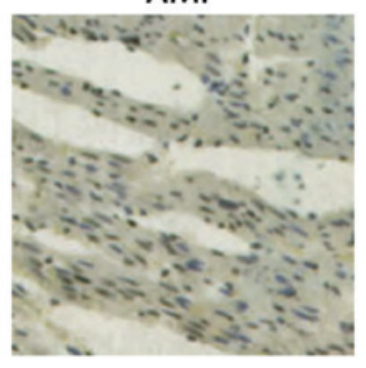

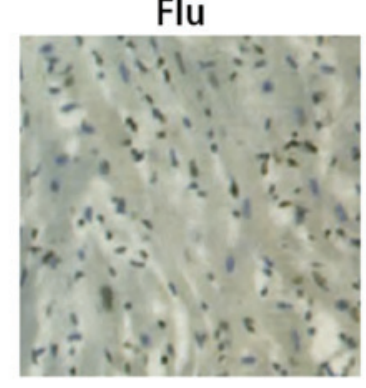

Flu

Flu

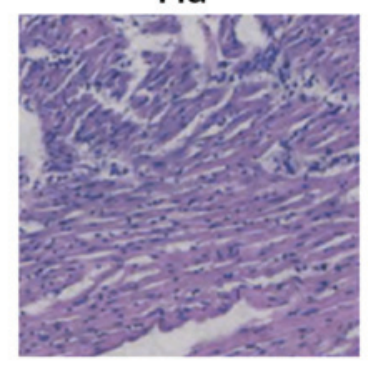

\section{C}

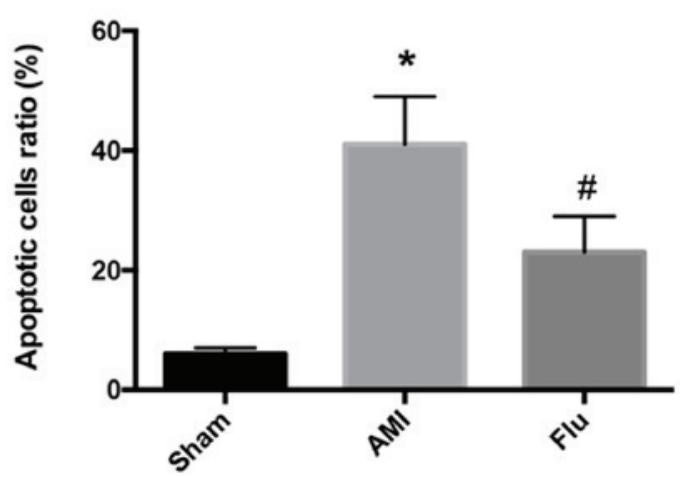

Figure 1. (A) H\&E staining results of myocardial tissues in infarction zones in Sham group, AMI group and Fluvastatin (Flu) group. (B) TUNEL staining results of myocardial tissues in infarction zones in Sham group, AMI group and Flu group, with brownish-yellow nuclei for positive cells. (C) Quantitative analysis results of apoptosis, expressed as mean \pm standard deviation, ${ }^{*} \mathrm{P}<0.05$ compared with Sham group, ${ }^{\sharp} \mathrm{P}<0.05$ compared with AMI group. H\&E, hematoxylin and eosin; Sham, sham operation; AMI, acute myocardial infarction.

Thereafter, sections were added with 50-100 $\mu$ 1 DAB substrate for reaction at $15-25^{\circ} \mathrm{C}$ for $10 \mathrm{~min}$. Then, they were counterstained with hematoxylin or methyl green for a few seconds and rinsed with tap water immediately, dehydrated with graded alcohol, permeabilized with xylene and mounted with neutral gum. A drop of PBS or glycerol was added, and the optical microscope was employed for counting (200-500 cells) and photography. Positive cells were counted, and apoptotic index $(\mathrm{AI})=$ number of positive cells $/$ total number of cells $\mathrm{x} 100$. The mean calculated in each group was used as a representative value.

Western blotting. Collected tissues were ground in liquid nitrogen, diluted with normal saline and placed on ice. The supernatant was collected and centrifuged at $12,000 \times$ g at $4^{\circ} \mathrm{C}$ for $5 \mathrm{~min}$, and the supernatant was discarded. The precipitate was re-suspended in radioimmunoprecipitation assay (RIPA) lysis buffer containing phenylmethylsulfonyl fluoride (PMSF) (Beyotime Institute of Biotechnology), lysed and centrifuged at $4^{\circ} \mathrm{C}$ and $16,000 \mathrm{x} \mathrm{g}$ for $15 \mathrm{~min}$, and the supernatant was taken for protein quantification. The protein was added with loading buffer, heated for denaturation, subjected to sodium dodecyl sulphate-polyacrylamide gel electrophoresis (SDS-PAGE) and transferred onto a membrane. The membrane was blocked with $5 \%$ skim milk for $2 \mathrm{~h}$, added with primary antibodies for incubation at $4^{\circ} \mathrm{C}$ overnight and washed with tris buffered saline-tween (TBST) 3 times (10 $\mathrm{min} /$ time), followed by incubation with corresponding secondary antibodies at room temperature for $1 \mathrm{~h}$ and washing with TBST 3 times $(10 \mathrm{~min} /$ time). Lastly, electrochemiluminescence (ECL) assay was performed to detect the protein expression in different samples.

Statistical analysis. Data were expressed as mean \pm standard deviation and analyzed through paired or unpaired t-test. Comparison between multiple groups was done using One-way ANOVA test followed by post hoc test (least significant difference). $\mathrm{P}<0.05$ indicates that the difference was statistically significant. Data were analyzed using the Statistical Product 
A

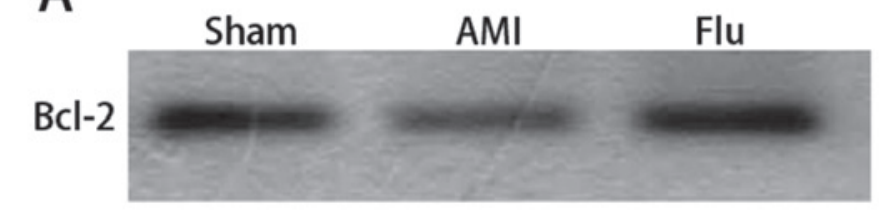

Bax
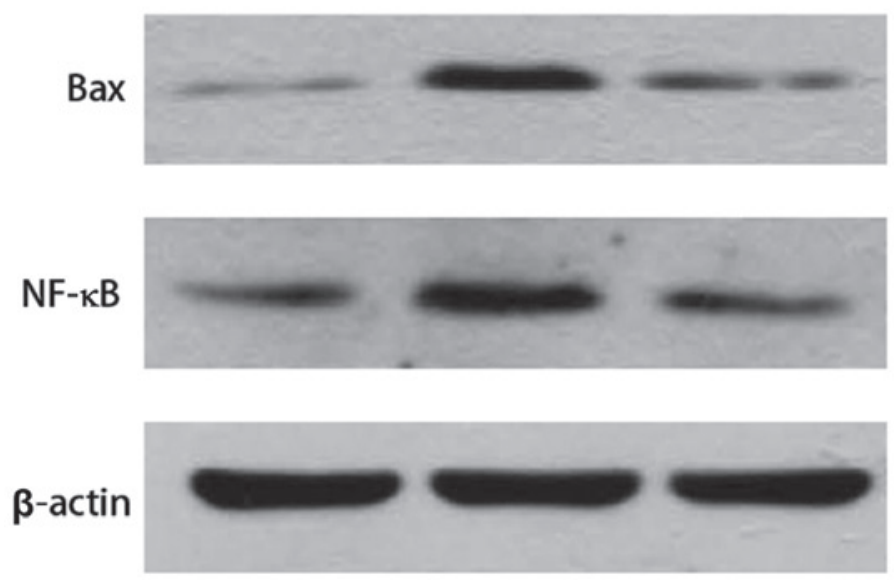

B
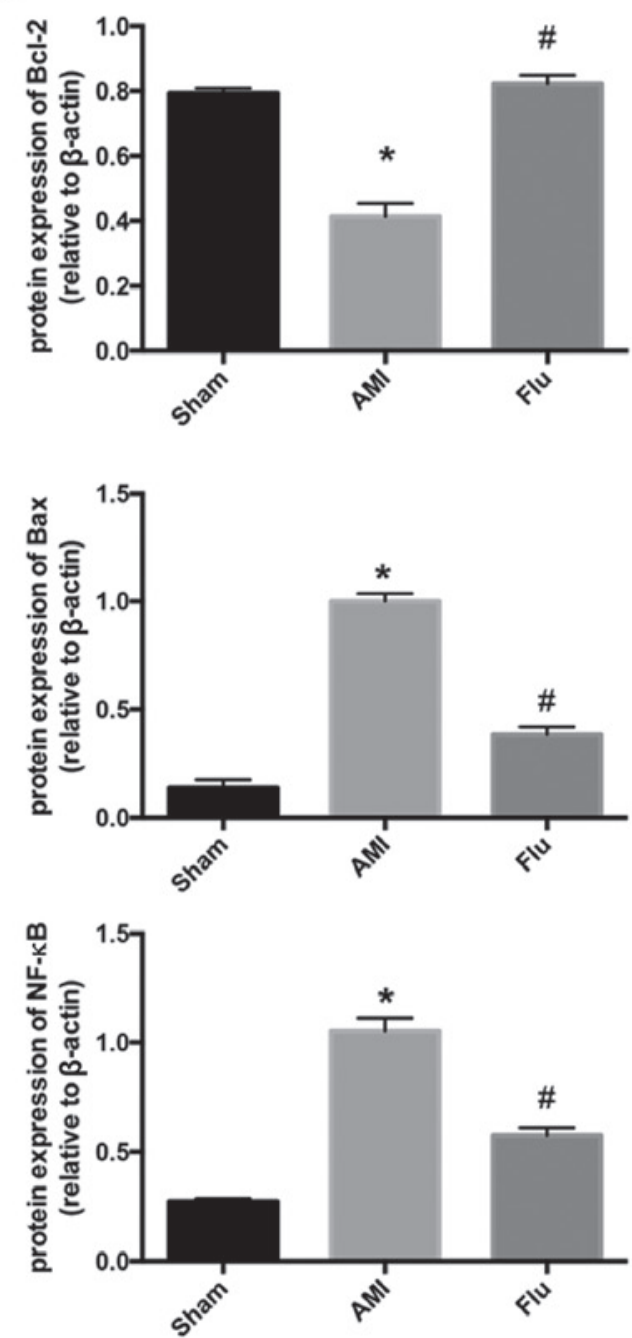

Figure 2. (A) Bcl-2, Bax and NF- $\kappa$ B protein content in infarction zones in Sham group, AMI group and Fluvastatin (Flu) group detected through western blotting. (B) Quantitatively-analyzed results of Bcl-2, Bax and NF- $\kappa$ B protein content in infarction zones in Sham group, AMI group and Flu group, expressed as mean \pm standard deviation, ${ }^{*} \mathrm{P}<0.05$ compared with Sham group, ${ }^{\#} \mathrm{P}<0.05$ compared with AMI group. Sham, sham operation; AMI, acute myocardial infarction; Bcl-2, B-cell lymphoma-2; Bax, Bcl-2 associated X protein; NF- $\mathrm{B}$, nuclear factor- $\kappa \mathrm{B}$.

and Service Solutions (SPSS) 20.0 statistical software (IBM), and then GraphPad software (GraphPad Software, Inc.) was used for plotting.

\section{Results}

Protective effect of Flu on myocardial cells in infarction region in AMI mice. After ischemic preconditioning, MI occurred in AMI group and Flu group, and it was alleviated in Flu group compared with that in AMI group (Fig. 1A). In AMI group, the level of antioxidant molecule superoxide dismutase (SOD) in serum dramatically declined compared with that in Sham group, while the level of oxidative damage marker malondialdehyde (MDA) was significantly higher than that in Sham group $(\mathrm{P}<0.05)$. In Flu group, the level of SOD was significant higher compared with that in AMI group, and the level of MDA was significantly lower than that in AMI group $(\mathrm{P}<0.05)$, indicating that Flu can exert an antioxidant effect in the protection of myocardial cells. In addition, there was no inflammatory cell infiltration, and the myocardial cells were arranged in an orderly manner in Sham group, AMI group had more inflammatory cell infiltration in the infarction region, disorderly myocardial fibers and fewer myocardial cells, and the inflammatory degree of myocardial cells was low and the myocardial cells were arranged in an orderly manner in Flu group (Fig. 1A). The above results suggest that the degree of myocardial tissue damage in AMI mice declines after Flu treatment.

Effect of Flu on myocardial apoptosis in infarction region in AMI mice. The results of terminal deoxynucleotidyl transferase-mediated dUTP nick end labeling (TUNEL) assay showed that the number of apoptotic myocardial cells was obviously increased in AMI group compared with that in Sham group, while it was obviously decreased in Flu group compared with that in AMI group (Fig. 1B and C). Compared with Sham group, AMI group had increased protein expression levels of Bax and nuclear factor $-\kappa \mathrm{B}(\mathrm{NF}-\kappa \mathrm{B})$, decreased protein expression of $\mathrm{Bcl}-2$ and increased $\mathrm{Bax} / \mathrm{Bcl}-2$ ratio in myocardial tissues. Compared with AMI group, Flu group 
A
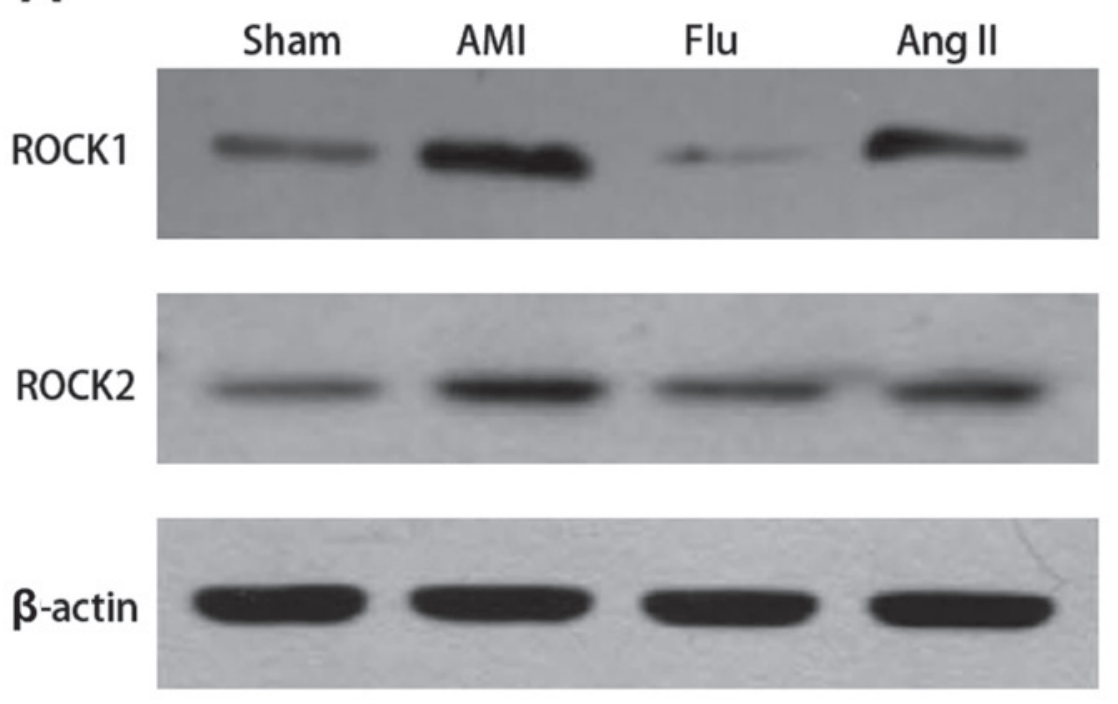

B
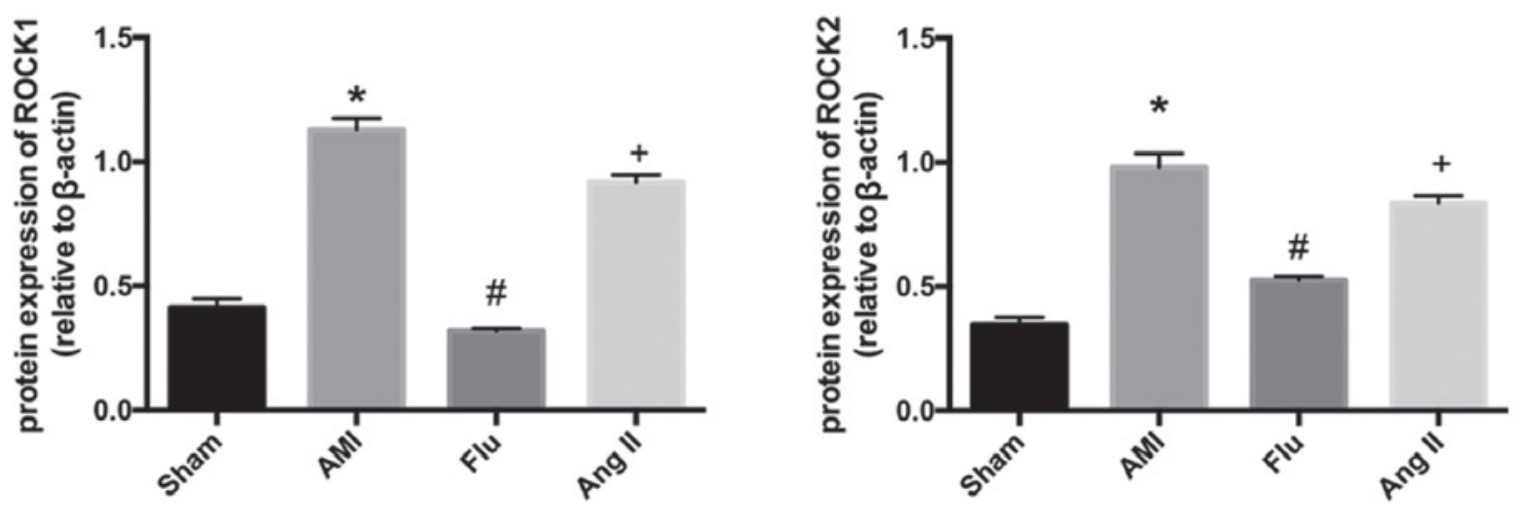

Figure 3. (A) ROCK1 and ROCK2 protein content in infarction zones in Sham group, AMI group, Fluvastatin (Flu) group and Flu+Ang II (Ang II) group detected through western blotting. (B) Quantitatively analyzed results of ROCK1 and ROCK2 protein content in infarction zones in Sham group, AMI group, Flu group and Flu+Ang II (Ang II) group expressed as mean \pm standard deviation, ${ }^{*} \mathrm{P}<0.05$ compared with Sham group, ${ }^{\sharp} \mathrm{P}<0.05$ compared with AMI group and ${ }^{+} \mathrm{P}<0.05$ compared with Flu group. Sham, sham operation; AMI, acute myocardial infarction; ROCK1, (Rho)-associated coiled-coil protein kinase 1; ROCK2, (Rho)-associated coiled-coil protein kinase 2; Ang II, Angiotensin II.

had decreased protein expression levels of Bax and $\mathrm{NF}-\kappa \mathrm{B}$, increased protein expression of Bcl-2 and decreased $\mathrm{Bax} / \mathrm{Bcl}-2$ ratio (Fig. $2 \mathrm{~A}$ and $\mathrm{B}$ ). The above results suggest that Flu can alleviate myocardial apoptosis in the infarction region in AMI mice.

Flu protects myocardial cells in infarction region in AMI mice through RhoA/ROCK pathway. The protein expression levels of ROCK1 and ROCK2 were evidently increased in AMI group compared with those in Sham group, while they were effectively inhibited by Flu (Fig. 3A and B). After treatment with the RhoA/ROCK agonist Ang II, the protein expression levels of ROCK1 and ROCK2 increased, the protein expression levels of Bax and NF- $\mathrm{BB}$ increased and the protein expression of Bcl-2 was decreased showing the mitigation effect of Flu on myocardial apoptosis in the infarction region in AMI mice was weakened. The results indicated that the Rho kinase may be the target of Flu (Figs. 3A and B, 4A and B).

\section{Discussion}

AMI is one of the common diseases seriously threatening human health. Myocardial dysfunction caused by myocardial apoptosis is the main cause of heart failure. Studies have shown that when MI occurs, myocardial apoptosis is increased, the Bax protein is upregulated and the $\mathrm{Bcl}-2$ protein is downregulated in the AMI animal model and humans (15-17). Therefore, inhibiting apoptosis may be an effective method to prevent MI (18-20). In the present study, the mouse model of AMI was established to confirm that exogenous injection of Flu can effectively alleviate ischemia-induced MI, inhibit pathological changes in myocardial tissues, reduce myocardial apoptosis 
A
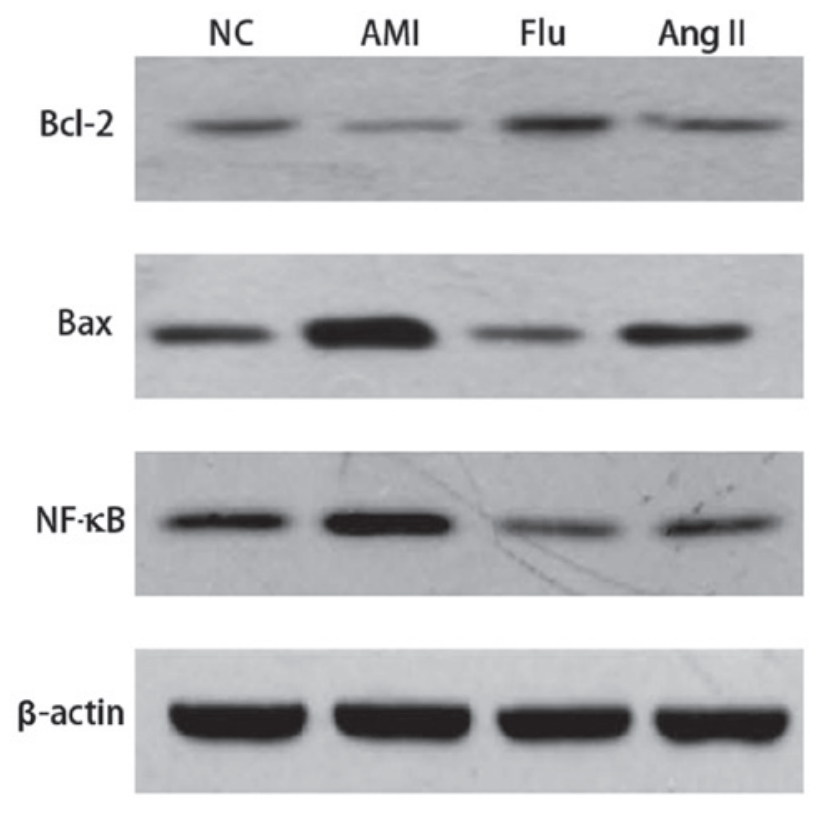

B
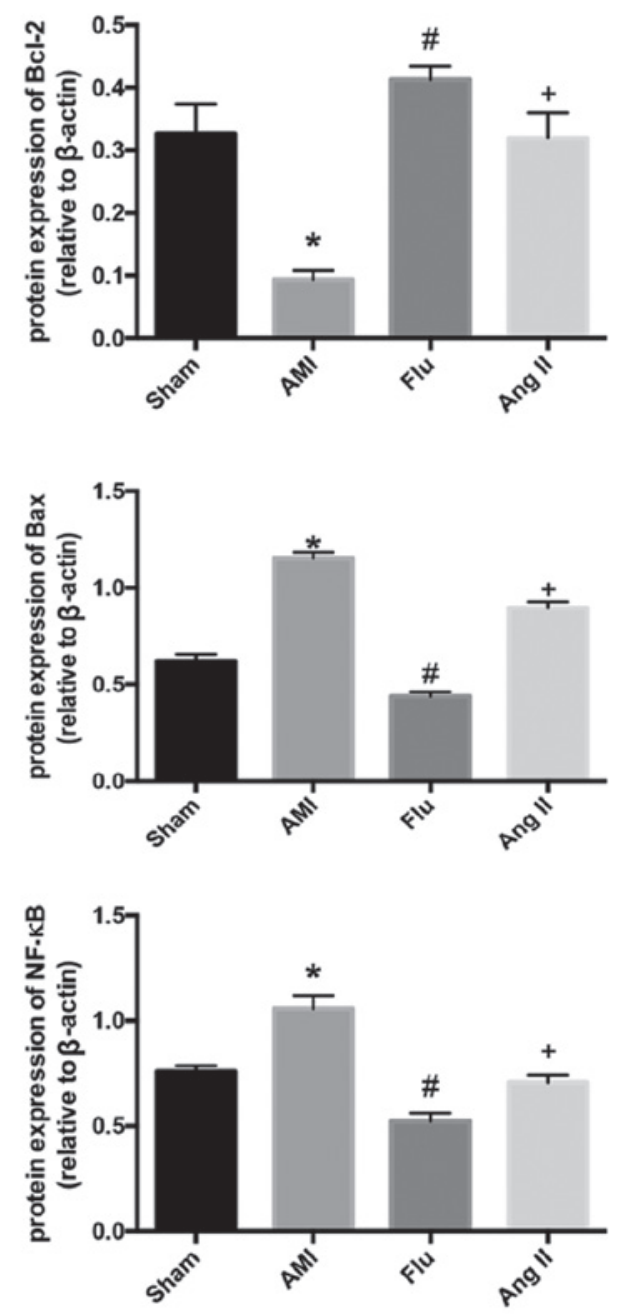

Figure 4. (A) Bcl-2, Bax and NF-kB protein content in infarction zones in Sham group, AMI group, Fluvastatin (Flu) group and Flu+Ang II (Ang II) group detected through western blotting. (B) Quantitatively-analyzed results of Bcl-2, Bax and NF-kB protein content in infarction zones in Sham group, AMI group, Flu group and Flu+Ang II (Ang II) group, expressed as mean \pm standard deviation, ${ }^{*} \mathrm{P}<0.05$ compared with Sham group, ${ }^{\text {}} \mathrm{P}<0.05$ compared with AMI group and ${ }^{+} \mathrm{P}<0.05$ compared with Flu group. Sham, sham operation; AMI, acute myocardial infarction; Bcl-2, B-cell lymphoma-2; Bax, Bcl-2 associated X protein;

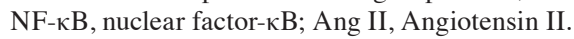

and increase Bcl-2 protein expression, which demonstrates that Flu can relieve ischemic-induced AMI through inhibiting myocardial apoptosis.

Myocardial apoptosis mediated by oxidative stress and inflammatory response, and decrease of viable myocardial cells are the major causes of heart failure in AMI patients. Mitochondrial dysfunction of ischemic myocardial cells leads to massive synthesis and secretion of reactive oxygen species (ROS), induces oxidative stress response in cells, alters mitochondrial membrane permeability and causes myocardial apoptosis (21-23). Inhibiting the expression of inflammatory factors in myocardial tissues can effectively alleviate AMI-induced cardiac dysfunction. In the present study, the expression levels of CRP, tumor necrosis factor- $\alpha$ (TNF- $\alpha$ ) and interleukin-6 (IL-6) in serum and ischemic myocardial tissues after Flu treatment were detected, and it was proven that Flu exerts a protective effect on ischemic myocardial tissues through reducing oxidative stress and inflammatory responses of myocardial tissues.
The Rho kinase belongs to the serine/threonine kinase of the small $\mathrm{G}$ protein family (protein kinase $\mathrm{A}, \mathrm{G}$ and $\mathrm{C}$ ), and it is involved in various physiological functions of cells through the downstream effector ROCK, which is considered to be related to myocardial apoptosis and MI (24-26). Studies have shown that after myocardial ischemia-reperfusion, the expression levels of RhoA and ROCK are upregulated, the phosphorylation of $\mathrm{I}-\kappa \mathrm{B}$ is enhanced, and NF- $\mathrm{KB}$ is activated and enters the nucleus to induce expression of inflammatory factors, thereby promoting inflammatory cell infiltration and adhesion. Inhibiting ROCK activity or knocking out the ROCK gene can effectively reduce the leukocyte recruitment and adhesion, and inhibit the level of various inflammatory factors $(27,28)$. ROCK is also involved in regulating the oxidative stress response, and inhibiting ROCK activation can lower the expression and activity of $\mathrm{NAD}(\mathrm{P}) \mathrm{H}$ in endothelial cells, and reduce the production of ROS (29). Rho kinase activation can upregulate the expression levels of TNF- $\alpha$ and IL-6, and its inhibitors have a protective effect 
on the myocardium (30). In addition, studies have manifested that apoptosis can be induced through a variety of pathways after Rho kinase is activated, such as exogenous myocardial apoptosis through regulating the TNF- $\alpha$ expression (16), and myocardial apoptosis by regulating the mitochondrial pathway (31) and through regulating caspase-3 activity (32). In this study, the expression levels of ROCK1 and ROCK2 as well as the key transcription factor for inflammatory response, $\mathrm{NF}-\kappa \mathrm{B}$, in myocardial tissues obviously declined after Flu treatment, suggesting that Flu exerts a protective effect against ischemia-induced AMI through regulating RhoA/ROCK.

In conclusion, the AMI model was established through acute myocardial ischemia in this study, to explore the effect of Flu on myocardial apoptosis and its protective mechanism in myocardial tissues. The results revealed that after Flu treatment, the MI severity and myocardial tissue lesions were effectively ameliorated in mice, the protein expression levels of Bax and NF- $\mathrm{KB}$ significantly declined, and the apoptotic myocardial cells were significantly reduced, whose mechanism may be that the activation of RhoA/ROCK signaling pathway is inhibited, thus suppressing the NF- $\mathrm{B}$-mediated inflammatory response, ROS-mediated oxidative stress and myocardial apoptosis. This study provides preclinical experimental support for the application of Flu and other statins in the prevention and treatment of AMI.

\section{Acknowledgements}

Not applicable.

\section{Funding}

No funding was received.

\section{Availability of data and materials}

All data generated or analyzed during this study are included in this published article.

\section{Authors' contributions}

ZY, JK and KC designed the study and performed the experiments, ZY and YW collected the data, JK and YW analyzed the data, ZY, JK and KC prepared the manuscript. All authors read and approved the final manuscript.

\section{Ethics approval and consent to participate}

The study was approved by the Animal Ethics Committee of Fujian Medical University Animal Center (Quanzhou, China).

\section{Patient consent for publication}

Not applicable.

\section{Competing interests}

The authors declare that they have no competing interests.

\section{References}

1. Shiny KS, Kumar SH, Farvin KH, Anandan R and Devadasan K: Protective effect of taurine on myocardial antioxidant status in isoprenaline-induced myocardial infarction in rats. J Pharm Pharmacol 57: 1313-1317, 2005.

2. Carson DA and Ribeiro JM: Apoptosis and disease. Lancet 341: 1251-1254, 1993

3. Wyllie AH, Kerr JF and Currie AR: Cell death: The significance of apoptosis. Int Rev Cytol 68: 251-306, 1980.

4. Yi J and An Y: Circulating miR-379 as a potential novel biomarker for diagnosis of acute myocardial infarction. Eur Rev Med Pharmacol Sci 22: 540-546, 2018.

5. Baldi A, Abbate A, Bussani R, Patti G, Melfi R, Angelini A, Dobrina A, Rossiello R, Silvestri F, Baldi F, et al: Apoptosis and post-infarction left ventricular remodeling. J Mol Cell Cardiol 34: $165-174,2002$

6. Slee EA, Harte MT, Kluck RM, Wolf BB, Casiano CA, Newmeyer DD, Wang HG, Reed JC, Nicholson DW, Alnemri ES, et al: Ordering the cytochrome c-initiated caspase cascade: Hierarchical activation of caspases-2, $-3,-6,-7,-8$, and -10 in a caspase-9-dependent manner. J Cell Biol 144: 281-292, 1999.

7. Sam F, Sawyer DB, Chang DL, Eberli FR, Ngoy S, Jain M, Amin J, Apstein CS and Colucci WS: Progressive left ventricular remodeling and apoptosis late after myocardial infarction in mouse heart. Am J Physiol Heart Circ Physiol 279: H422-H428, 2000.

8. Wu J, Guo Z, Wang LL and Zhang RL: Degeneration of sensory afferent nerves enhances pulmonary inflammatory alterations in acute myocardial infarction in rats. Cardiovasc Pathol 21: 149-157, 2012.

9. Youle RJ and Strasser A: The BCL-2 protein family: Opposing activities that mediate cell death. Nat Rev Mol Cell Biol 9: 47-59, 2008.

10. Burridge $\mathrm{K}$ and Wennerberg K: Rho and Rac take center stage. Cell 116: 167-179, 2004

11. Etienne-Manneville S and Hall A: Rho GTPases in cell biology. Nature 420: 629-635, 2002.

12. Shimokawa H: Rho-kinase as a novel therapeutic target in treatment of cardiovascular diseases. J Cardiovasc Pharmacol 39: 319-327, 2002.

13. Yamakawa T, Tanaka S, Kamei J, Kadonosono K and Okuda K: Pitavastatin inhibits vascular smooth muscle cell proliferation by inactivating extracellular signal-regulated kinases $1 / 2$. J Atheroscler Thromb 10: 37-42, 2003.

14. Wiesbauer F, Kaun C, Zorn G, Maurer G, Huber K and Wojta J: HMG CoA reductase inhibitors affect the fibrinolytic system of human vascular cells in vitro: A comparative study using different statins. Br J Pharmacol 135: 284-292, 2002.

15. Kitano K, Usui S, Ootsuji H, Takashima S, Kobayashi D, Murai H, Furusho H, Nomura A, Kaneko S and Takamura M: Rho-kinase activation in leukocytes plays a pivotal role in myocardial ischemia/reperfusion injury. PLoS One 9: e92242, 2014.

16. Chang CC, Wang SS, Hsieh HG, Lee WS, Chuang CL, Lin HC, Lee FY, Lee SD and Huang HC: Rosuvastatin improves hepatopulmonary syndrome through inhibition of inflammatory angiogenesis of lung. Clin Sci (Lond) 129: 449-460, 2015.

17. Wang Y, Zhang H, Chai F, Liu X and Berk M: The effects of escitalopram on myocardial apoptosis and the expression of Bax and Bcl-2 during myocardial ischemia/reperfusion in a model of rats with depression. BMC Psychiatry 14: 349, 2014.

18. Chen TL, Zhu GL, He XL, Wang JA, Wang Y and Qi GA: Short-term pretreatment with atorvastatin attenuates left ventricular dysfunction, reduces infarct size and apoptosis in acute myocardial infarction rats. Int J Clin Exp Med 7: 4799-4808, 2014.

19. Malick M, Gilbert K, Barry M, Godbout R and Rousseau G: Desvenlafaxine reduces apoptosis in amygdala after myocardial infarction. Brain Res Bull 109: 158-163, 2014.

20. Liu Q: Lentivirus mediated interference of Caspase-3 expression ameliorates the heart function on rats with acute myocardial infarction. Eur Rev Med Pharmacol Sci 18: 1852-1858, 2014.

21. Kumphune S, Surinkaew S, Chattipakorn SC and Chattipakorn N: Inhibition of $\mathrm{p} 38$ MAPK activation protects cardiac mitochondria from ischemia/reperfusion injury. Pharm Biol 53: 1831-1841, 2015.

22. Motloch LJ, Hu J and Akar FG: The mitochondrial translocator protein and arrhythmogenesis in ischemic heart disease. Oxid Med Cell Longev 2015: 234104, 2015. 
23. Zhang Q, Wang H, Yang YJ, Dong QT, Wang TJ, Qian HY, Li N, Wang XM and Jin C: Atorvastatin treatment improves the effects of mesenchymal stem cell transplantation on acute myocardial infarction: The role of the RhoA/ROCK/ERK pathway. Int J Cardiol 176: 670-679, 2014.

24. Loirand G, Guérin P and Pacaud P: Rho kinases in cardiovascular physiology and pathophysiology. Circ Res 98: 322-334, 2006.

25. Shi J and Wei L: Rho kinases in cardiovascular physiology and pathophysiology: The effect of fasudil. J Cardiovasc Pharmacol 62: 341-354, 2013.

26. Hung CN, Huang HP, Wang CJ, Liu KL and Lii CK: Sulforaphane inhibits TNF- $\alpha$-induced adhesion molecule expression through the Rho A/ROCK/NF- $\mathrm{KB}$ signaling pathway. J Med Food 17: 1095-1102, 2014

27. Bao W, Hu E, Tao L, Boyce R, Mirabile R, Thudium DT, Ma XL, Willette RN and Yue TL: Inhibition of Rho-kinase protects the heart against ischemia/reperfusion injury. Cardiovasc Res 61: $548-558,2004$

28. Shimokawa H and Takeshita A: Rho-kinase is an important therapeutic target in cardiovascular medicine. Arterioscler Thromb Vasc Biol 25: 1767-1775, 2005.

29. Higashi M, Shimokawa H, Hattori T, Hiroki J, Mukai Y, Morikawa K, Ichiki T, Takahashi S and Takeshita A: Long-term inhibition of Rho-kinase suppresses angiotensin II-induced cardiovascular hypertrophy in rats in vivo: Effect on endothelial NAD(P)H oxidase system. Circ Res 93: 767-775, 2003.
30. Petrache I, Crow MT, Neuss $M$ and Garcia JG: Central involvement of Rho family GTPases in TNF-alpha-mediated bovine pulmonary endothelial cell apoptosis. Biochem Biophys Res Commun 306: 244-249, 2003.

31. Del Re DP, Miyamoto S and Brown JH: RhoA/Rho kinase up-regulate Bax to activate a mitochondrial death pathway and induce cardiomyocyte apoptosis. J Biol Chem 282: 8069-8078, 2007.

32. Chang J, Xie M, Shah VR, Schneider MD, Entman ML, Wei L and Schwartz RJ: Activation of Rho-associated coiled-coil protein kinase 1 (ROCK-1) by caspase-3 cleavage plays an essential role in cardiac myocyte apoptosis. Proc Natl Acad Sci USA 103: 14495-14500, 2006. International (CC BY-NC-ND 4.0) License. 